\title{
COMMENTARY
}

\section{Antibiotics for the critically ill: more than just selecting appropriate initial therapy}

\author{
Marin H Kollef* \\ See related research by Carlier et al., http://ccforum.com/content/17/3/R84
}

\begin{abstract}
Critically ill patients with infection provide a number of challenges to clinicians in terms of optimizing their antimicrobial treatment. Of foremost importance, initial antibiotic treatment should be selected as to provide coverage for the causative pathogens. However, the administration of those antibiotics (dosing, interval of administration, duration of infusion, route of administration) should be prescribed in a manner to ensure optimal drug delivery to the site of infection. This is a challenge given the characteristics of many infected critically ill patients (shock, elevated cardiac output in the resuscitated state, supranormal creatinine clearance, increased volume of distribution). Intensive care unit practitioners should utilize treatment strategies that strive to deliver antibiotics in an individualized manner aimed at attaining desired pharmacokinetic/pharmacodynamic targets. The goal of such a treatment strategy is to maximize the likelihood of curing the infection and allowing the critically ill patient the best opportunity for recovery. Effective implementation of antimicrobial optimization delivery strategies will likely require a multi-disciplinary approach including intensivists, pharmacists, and infectious disease specialists.
\end{abstract}

In this issue of Critical Care, Carlier and colleagues describe pharmacokinetic/pharmacodynamic (PK/PD) target attainment in critically ill patients receiving meropenem or piperacillin/tazobactam as extended infusions [1]. These investigators found that $48 \%$ of patients did not achieve the desired PK/PD target (100\% time that the free antibiotic fraction exceeds the minimum inhibitory concentration (MIC)), of which almost $80 \%$ had a measured

*Correspondence: mkollef@dom.wustl.edu

Division of Pulmonary and Critical Care Medicine, Washington University School of Medicine, 660 South Euclid Avenue, Campus Box 8052, St Louis, MO 63110, USA creatinine clearance $(\mathrm{CrCl})>130 \mathrm{ml} /$ minute. More worrisome was the observation that $37 \%$ of patients with $\mathrm{CrCl}>130 \mathrm{ml} /$ minute did not achieve the minimum PK/ PD target (50\% time that the free antibiotic fraction exceeds the MIC). These investigators concluded that patients not attaining PK/PD targets may be at risk for treatment failure without upward antibiotic dose titration. The findings from this study suggest that we should add inadequate antibiotic dosing to the list of antibiotic treatment characteristics that can adversely impact the outcomes of infected critically ill patients. This is an important issue when one considers that antibiotic use is often viewed as a variable expense in the hospital setting that can be manipulated to achieve cost savings. Such an attitude is flawed with the understanding that the most cost-effective approach to treating serious infections is the approach that is most likely to achieve rapid clinical success.

The most recent Surviving Sepsis Campaign Guidelines recommend that broad-spectrum antibiotics be administered within the first hour of recognition of septic shock, with one or more agents that have activity against the likely causative pathogens, and that the duration of therapy typically be between 7 and 10 days [2]. These recommendations are based on studies demonstrating that the timing of appropriate antibiotic therapy, defined as an antimicrobial regimen demonstrating in vitro activity against the isolated organism(s) responsible for the infection, is critical in determining the outcomes of such patients [3-5]. However, even if an appropriate initial therapy with an active antibiotic regimen is administered, patient outcomes may not be optimized due to inadequate drug concentration delivery to the site of infection. This is important for both concentrationdependent antibiotics as well as for antibiotics whose efficacy is based on the achieved dosing interval time that the antibiotic concentration is above the MIC of the pathogen. Moreover, many prescribers of antimicrobial therapy in the hospitalized patient are unaware that drug delivery targets are not met given that therapeutic drug monitoring is not routinely performed for most antibiotic classes. 
Recent randomized trials of inadequately dosed antibiotics (ceftobiprole and tigecycline) for patients with nosocomial pneumonia have demonstrated greater treatment failures and mortality for the inadequately dosed antibiotics compared to more optimally dosed comparators [6-8]. The usual rationale for selecting such doses for investigational antibiotics is to minimize any drug-related toxicity. This is highlighted by the current dosing of tigecycline that is half of the originally considered dosing due to greater rates of nausea and vomiting at the higher doses (the higher doses being more likely to achieve desired drug concentration PK/PD targets). Additionally, the results from two meta-analyses and a recent clinical trial found that the use of prolonged infusions of betalactam antibiotics achieved similar clinical results compared to similar or higher dosed intermittent infusion antibiotic therapy [9-11]. However, in one of the metaanalyses, a trend towards benefit among patients receiving intermittent infusion antibiotics possibly explained by the use of higher antibiotic doses was observed [10]. For fair balance it should be noted that another recent meta-analysis showed that prolonged or continuous infusion of pipercillin-tazobactam or carbapenems was associated with lower mortality, although these results were primarily from nonrandomized studies [12].

The influence of $\mathrm{CrCl}$ on outcomes among patients with severe infections also seems to be an important determinant of clinical outcome, in large part by influencing antibiotic elimination and achieved drug concentration targets at the infection site. Elevated $\mathrm{CrCl}$ has been shown to be an important predictor of subtherapeutic betalactam concentrations $[13,14]$. The use of prolonged antibiotic infusions has been proposed as a strategy to achieve appropriate targeted antibiotic blood concentrations in patients with augmented renal clearance [15]. The potential detrimental influence of augmented $\mathrm{CrCl}$ on the outcomes of critically ill patients with infections has been demonstrated in several randomized trials. Kollef and colleagues [16] found that patients with $\mathrm{CrCl} \geq 150 \mathrm{ml} /$ minute had greater clinical cure rates for VAP with 10 days of imipenem compared to 7 days of meropenem despite administering meropenem as a prolonged infusion. In a trial of nosocomial pneumonia, ceftobiprole dosed at $500 \mathrm{mg}$ every 8 hours was clinically inferior and associated with greater mortality compared to ceftazidime dosed at 2 grams every 8 hours plus linezolid dosed at $600 \mathrm{mg}$ every 12 hours [8]. In this trial, increased $\mathrm{CrCl}$ was found to be associated with a greater risk of mortality, suggesting that under-dosing of antibiotics may be most detrimental in patients with normal or augmented $\mathrm{CrCl}$. This may also explain the hesitation among intensivists to use the currently approved dosing regimen of ceftaroline (600 mg every 12 hours) in critically ill patients as it has not been rigorously evaluated in that population.
Carbapenem antibiotics and beta-lactams represent the most common antibiotics currently prescribed in critically ill patients. However, there is no routine therapeutic drug monitoring available for these agents as exists for aminoglycosides and vancomycin. This creates uncertainty for many clinicians prescribing antibiotics, especially when patient-specific conditions such as renal function, volume status, and hemodynamics are changing. Therefore, intensivists must carefully consider how antibiotics are delivered in terms of dose, interval of administration, and duration of infusion in order to optimize PK/PD target attainment. The duration of therapy must also be carefully considered in that longer durations of treatment for optimal success may be necessary for difficult to treat pathogens such as Pseudomonas or Acinetobacter species [16]. A recent example of new methods for achieving such targets is the use of advanced aerosol delivery systems that hold the promise of exceeding therapeutic targets in the lung with antibiotics such as colistin, aminoglycosides, and fosfomycin that cannot be accomplished with parenteral administration.

\section{Conclusion}

Carlier and colleagues must be commended for their study highlighting the relationship between elevated $\mathrm{CrCl}$ and inability to attain PK/PD targets for prescribed antibiotics despite the use of prolonged infusions. This is an important finding given the increasing use of prolonged antibiotic infusions and suggests that clinicians should carefully evaluate the presence of elevated $\mathrm{CrCl}$ when making decisions regarding antibiotic dosing. From a practical standpoint, the use of an antibiotic loading dose along with the prolonged antibiotic infusion should be considered in patients with elevated $\mathrm{CrCl}$ to allow for more rapid attainment of the desired antibiotic concentration at the infection site. Moreover, maximal recommended doses of antibiotics should be prescribed in critically ill patients, especially in the presence of elevated $\mathrm{CrCl}$, as suggested by Arnold and colleagues [11] regardless of whether or not prolonged infusions are employed (for example, cefepime 2 grams every 8 hours, pipercillintazobactam 4.5 grams every 6 hours, meropenem 1 gram every 8 hours). Future studies are needed that address the issue of antibiotic dosing optimization in the presence or absence of elevated $\mathrm{CrCl}$. Such studies also need to carefully consider the influence of confounding factors, such as the MIC of the targeted pathogens and the patient's drug volume of distribution, that can also influence the optimization of antibiotic dosing.

\footnotetext{
Abbreviations

$\mathrm{CrCl}$, creatinine clearance; $\mathrm{MIC}$, minimum inhibitory concentration; $\mathrm{PK} / \mathrm{PD}$ pharmacokinetic/pharmacodynamic.
}

Competing interests

The author declares that they have no competing interests. 
Author's contribution

MHK was solely responsible for the development and writing of this article.

\section{Acknowledgements}

Dr Kollef's efforts are supported by the Barnes-Jewish Hospital Foundation.

Published: 23 May 2013

\section{References}

1. Carlier M, Carrette S, Roberts JA, Stove V, Verstraete AG, Hoste E, Depuydt P, Decruyenaere J, Wallis SC, Lipman J, DeWaele JJ: Meropenem and piperacillin/tazobactam prescribing in critically ill patients: does augmented renal clearance affect pharmacokinetic/pharmacodynamic target attainment when extended infusions are used? Crit Care 2013, 17:R84.

2. Dellinger RP, Levy MM, Rhodes A, Annane D, Gerlach H, Opal SM, Sevransky JE, Sprung CL, Douglas IS, Jaeschke R, Osborn TM, Nunnally ME, Townsend SR, Reinhart K, Kleinpell RM, Angus DC, Deutschman CS, Machado FR, Rubenfeld GD, Webb SA, Beale RJ, Vincent JL, Moreno R; Surviving Sepsis Campaign Guidelines Committee including the Pediatric Subgroup: Surviving sepsis campaign: international guidelines for management of severe sepsis and septic shock: 2012. Crit Care Med 2013, 41:580-637.

3. Kollef MH, Sherman G, Ward S, Fraser VJ: Inadequate antimicrobial treatment of infections: a risk factor for hospital mortality among critically ill patients. Chest 1999, 115:462-474.

4. Kumar A, Roberts D, Wood KE, Light B, Parrillo JE, Sharma S, Suppes R, Feinstein D, Zanotti S, Taiberg L, Gurka D, Kumar A, Cheang M: Duration of hypotension before initiation of effective antimicrobial therapy is the critical determinant of survival in human septic shock. Crit Care Med 2006, 34:1589-1596.

5. Kollef M, Micek S, Hampton N, Doherty JA, Kumar A: Septic shock attributed to Candida infection: importance of empiric therapy and source control. Clin Infect Dis 2012, 54:1739-1746.

6. Freire AT, Melnyk V, Kim MJ, Datsenko O, Dzyublik O, Glumcher F, Chuang YC, Maroko RT, Dukart G, Cooper CA, Korth-Bradley JM, Dartois N, Gandjini H; 311 Study Group: Comparison of tigecycline with imipenem/cilastatin for the treatment of hospital-acquired pneumonia. Diagn Microbiol Infect Dis 2010, 68:140-151.

7. Prasad P, Sun J, Danner RL, Natanson C: Excess deaths associated with tigecycline after approval based on noninferiority trials. Clin Infect Dis 2012, 54:1699-1709.
8. Basilea Announces Positive Top-line Data from Phase III Study of Ceftobiprole in Hospital-Acquired Pneumonia

[http://www.basilea.com/News-and-Media/ Basilea-Announces-Positive-Top-line-Data-from-Phase-III-Study-ofCeftobiprole-in-Hospital-Acquired-Pneumonia/144]

9. Tamma PD, Putcha N, Suh YD, Van Arendonk KJ, Rinke ML: Does prolonged $\beta$-lactam infusions improve clinical outcomes compared to intermittent infusions? A meta-analysis and systematic review of randomized, controlled trials. BMC Infect Dis 2011, 11:181.

10. Roberts JA, Webb S, Paterson D, Ho KM, Lipman J: A systematic review on clinical benefits of continuous administration of beta-lactam antibiotics. Crit Care Med 2009, 37:2071-2078.

11. Arnold HM, Hollands JM, Skrupky LP, Smith JR, Juang PH, Hampton NB, McCormick S, Reichley RM, Hoban A, Hoffmann J, Micek ST, Kollef MH: Prolonged infusion antibiotics for suspected gram-negative infections in the ICU: a before-after study. Ann Pharmacother 2013, 47:170-180.

12. Falagas ME, Tansarli GS, Ikawa K, Vardakas KZ: Clinical outcomes with extended or continuous versus short-term intravenous infusion of carbapenems and piperacillin/tazobactam: a systematic review and metaanalysis. Clin Infect Dis 2013, 56:272-282.

13. Udy AA, Varghese JM, Altukroni M, Briscoe S, McWhinney BC, Ungerer JP, Lipman J, Roberts JA: Subtherapeutic initial $\beta$-lactam concentrations in select critically ill patients: association between augmented renal clearance and low trough drug concentrations. Chest 2012, 142:30-39.

14. Baptista JP, Sousa E, Martins PJ, Pimentel JM: Augmented renal clearance in septic patients and implications for vancomycin optimisation. Int J Antimicrob Agents 2012, 39:420-423.

15. Roberts JA, Lipman J: Optimal doripenem dosing simulations in critically ill nosocomial pneumonia patients with obesity, augmented renal clearance, and decreased bacterial susceptibility. Crit Care Med 2013, 41:489-495.

16. Kollef MH, Chastre J, Clavel M, Restrepo MI, Michiels B, Kaniga K, Cirillo I, Kimko H, Redman R: A randomized trial of 7-day doripenem versus 10-day imipenem-cilastatin for ventilator-associated pneumonia. Crit Care 2012, 16:R218.

doi:10.1186/cc12698

Cite this article as: Kollef $\mathrm{MH}$ : Antibiotics for the critically ill: more than just selecting appropriate initial therapy. Critical Care 2013, 17:146. 